\title{
CONVERGÊNCIA DOS MODELOS DE ÁRVORES BINOMIAIS PARA AVALIAÇÃO DE OPÇÕES
}

\author{
Tara Keshar Nanda Baidya \\ Departamento de Engenharia Industrial - PUC-Rio
}

\author{
Alessandro de Lima Castro \\ Centro de Pesquisas de Energia Elétrica - CEPEL e \\ Departamento de Engenharia Industrial - PUC-Rio
}

\begin{abstract}
Resumo
Black \& Scholes (1973) desenvolveram um modelo para a avaliação de opções de compra e venda do tipo Europeu. Merton (1973) estendeu o modelo para ações que pagam dividendos. Muitos outros desenvolvimentos foram feitos acerca dos dois trabalhos citados, mas talvez um dos mais importantes foi proposto por Cox, Ross \& Rubinstein (1979), onde o processo estocástico (para o preço da ação objeto) em tempo e estado contínuo (Movimento Geométrico Browniano) proposto por Black \& Scholes foi aproximado por um processo de tempo e estado discreto (Random Walk). O modelo de Cox, Ross \& Rubinstein, hoje conhecido como Modelo Binomial, tornou-se um dos métodos mais utilizados para calcular o valor de opções, principalmente opções americanas, devido a sua simplicidade e fácil implementação computacional. Mas, o modelo binomial possui uma convergência fraca, em forma oscilatória, para o valor verdadeiro. Este artigo pretende mostrar as principais soluções encontradas na literatura para acelerar a convergência.
\end{abstract}

Palavras-chave: engenharia econômica; modelo binomial; convergência oscilatória.

\begin{abstract}
Black \& Scholes (1973) developed a model for pricing European call options on assets that do not pay dividends. Merton (1973) extended it to include assets that pay dividends. Many other developments have been made after that. Perhaps one of the most important work in this area was proposed by Cox, Ross \& Rubinstein (1979), where the stochastic process for the price of the underlying asset proposed by Black \& Scholes was approximated by a discrete time binomial process. The method proposed by Cox, Ross \& Rubinstein became very popular because of its simplicity and easy implementation. But the convergence of the binomial model is weak and oscillatory. This work pretends to explain the main solutions found in the literature to accelerate convergence.
\end{abstract}

Keywords: engineering economics; binomial option pricing; oscillatory convergence. 


\section{Introdução}

Em 1973, Black Fischer e Myron Scholes desenvolveram um modelo para avaliação de opções de compra do tipo européia. Eles partiram do pressuposto que o preço de uma ação segue um processo estocástico conhecido como Movimento Geométrico Browniano. A equação que rege este processo é dada por:

$$
d S=r S d t+\sigma S d z
$$

onde $S$ é o preço do ativo objeto, $r$ é a taxa de juros livre de risco, $\sigma$ é o desvio padrão do preço do ativo objeto e $d \mathrm{z}$ é o incremento de um processo de Wiener, com média 0 e desvio padrão $d$ t. Os parâmetros $r$ e $\sigma$ são considerados constantes ao longo do tempo.

Da suposição sobre o comportamento do preço da ação, o processo que o título derivativo segue pode ser deduzido usando a idéia chave do modelo de Black e Scholes que é a formação de uma carteira dinâmica livre de risco, resultando em uma equação diferencial parcial, dada a seguir:

$$
\frac{1}{2} \sigma^{2} S^{2} \frac{\partial^{2} C}{\partial S^{2}}+r S \frac{\partial C}{\partial S}+\frac{\partial C}{\partial t}-r C=0
$$

Onde $C$ é o valor da opção. Ao ser resolvida a equação (2), foi encontrada a seguinte fórmula que calcula o preço de uma opção de compra do tipo Européia:

$$
C=S N\left(d_{1}\right)-X e^{r(T-t)} N\left(d_{2}\right)
$$

onde

$$
\begin{aligned}
& d_{1}=\frac{\ln \left(\frac{S}{X}\right)+\left(r+\frac{1}{2} \sigma^{2}\right)(T-t)}{\sigma \sqrt{T-t}} \\
& d_{2}=d_{1}-\sigma \sqrt{T-t}
\end{aligned}
$$

$N($.) é a função de distribuição normal cumulativa, $X$ é o preço de exercício da opção e T é tempo de maturação da opção. Muitos trabalhos baseados na metodologia de Black e Scholes surgiram em seguida, entre os mais importantes podemos citar:

- Merton (1973) estendeu o modelo de Black e Scholes para ações com pagamento de dividendos e mostrou que uma opção de compra americana, onde a ação objeto não paga dividendos, pode ser avaliada como se fosse uma opção de compra européia, ou seja, o exercício antecipado nunca será ótimo;

- Cox \& Ross (1976) estenderam o modelo de Black e Scholes para ativos objeto que seguiam outros processos estocásticos que não o Movimento Geométrico Browniano, como por exemplo, Poisson.

Ainda existia uma questão como avaliar uma opção Americana que pode ser exercida antecipadamente? Nenhuma solução analítica fora encontrada, então métodos numéricos ou de aproximação deveriam ser utilizados. Brennan \& Schwartz (1978) aplicaram métodos de diferença finita para resolver a equação diferencial parcial para uma opção americana.

Diferentemente, Cox, Ross \& Rubinstein (1979) utilizaram um processo discreto no tempo e binomial no espaço para aproximar um processo contínuo e calcular o preço de uma opção Americana. Devido a simplicidade, a facilidade de implementação e principalmente a flexibilidade, o modelo de árvores binomiais (como ficou conhecido) tornou-se uma das metodologias mais utilizadas para a avaliação de opções Americanas. 
Desde 1979 até hoje muitos desenvolvimentos foram feitos a partir do modelo binomial tornando-o ainda mais abrangente e também podendo modelar opções onde a taxa de juros e/ou volatilidade são variantes com o tempo, os dividendos podem ser contínuos, proporcionais ao preço da ação ou valores discretos.

Mais recentemente a comunidade acadêmica e o mercado têm-se preocupado com o tempo de processamento de diversos métodos numéricos usados em finanças. Existe a necessidade que esses métodos sejam rápidos e precisos. É claro que nem sempre é possível encontrar métodos numéricos que possuam essas características. O modelo binomial, por ser uma aproximação discreta de um evento em tempo contínuo, é considerado um método numérico e sofre dessas restrições. Além disso, possui um problema grave: a convergência oscilatória. Vários pesquisadores têm estudado o modelo binomial de modo a eliminar os efeitos da oscilação, mantendo-o simples de entender, fácil de implementar e flexível na hora ser utilizado, ou seja, existe um esforço para torná-lo mais rápido em sua convergência para o valor verdadeiro sem que sejam perdidas suas principais características.

Este trabalho têm como objetivo principal mostrar alguns aprimoramentos sugeridos na literatura nos últimos anos para melhorar a convergência do modelo binomial. Serão apresentados modelos que diminuem o efeito da convergência oscilatória.

O restante do artigo será estruturado da seguinte forma: na seção 2 serão apresentados os principais modelos para os parâmetros das árvores binomiais; a seção 3 mostrará como uma árvore binomial pode ser utilizada para calcular o preço de opções Americanas; a seção 4 mostrará as principais técnicas utilizadas para diminuir os efeitos da convergência oscilatória nos modelos binomiais e a seção 5 apresentará as principais conclusões.

\section{Modelos de Árvores Binomiais}

Cox, Ross \& Rubinstein (1979) desenvolveram um método que converge para a solução encontrada por Black \& Scholes (1973). Ao invés de resolver a equação (2) utilizando método numéricos, como Brennan \& Schwartz (1978), eles mostraram que a equação (1) poderia ser obtida como um limite contínuo de um caminho aleatório em tempo discreto. Para isso, o tempo deve ser dividido em períodos discretos de comprimento $\Delta t$ e será assumido que em cada período, o preço da ação objeto $S$ ou move-se para cima ou para baixo segundo as constantes proporcionais $u$ e $d$, respectivamente. A probabilidade de mover para cima é dada por uma medida neutra ao risco $p$ e para baixo por $q=1-p$. A Figura 1 mostra os possíveis valores de $S$ ao longo de três intervalos de tempo.

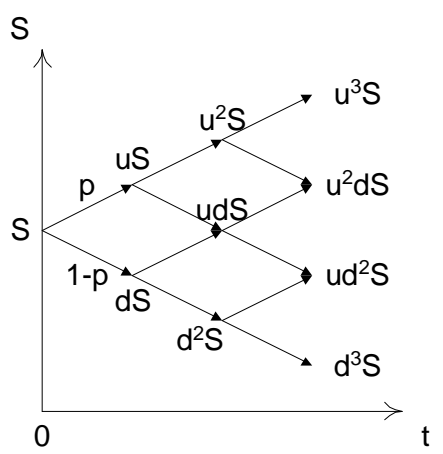

Figura 1 - Árvore Binomial com três períodos 
Esta árvore é conhecida como árvore binomial multiplicativa, pois os possíveis valores futuros em cada período são distribuídos segundo a distribuição binomial e são proporcionais ao estado inicial. Os modelos que serão mostrados a seguir têm como objetivo calcular os parâmetros da árvores, $u, d$ e $p$.

\subsection{Modelo de Cox-Ross-Rubinstein (CRR)}

Considere a equação (1) novamente. Esta equação será ligeiramente modificada para levar em consideração os dividendos pagos pelo ativo objeto. Seja $\delta$ uma taxa de dividendos por unidade de tempo, então a equação diferencial estocástica que modela os movimentos nos preços do ativo objeto pode ser rescrita como:

$$
d S=(r-\delta) S d t+\sigma S d z
$$

Agora, divida a equação (5) por $S$, obtendo $d S / S=(r-\delta) d t+\sigma d z$. Substitua a variável $S$ por $x$ usando a relação $x=\ln S$. Para isso, basta derivar $x$ em relação a $S$, $d x / d S=1 / S \Rightarrow d x=d S / S$. Assim, a equação (1) pode ser rescrita

$$
d x=(r-\delta) d t+\sigma d z
$$

Por questão de comodidade faça

$$
v=r-\delta
$$

Tomando-se variações discretas de $x$ e $t$, a equação (6) pode ser escrita da seguinte forma:

$$
\Delta x=v \Delta t+\sigma \Delta z
$$

onde $\Delta t=T / n, T$ é a vida útil da opção e $n$ é o número de intervalos de tempo. $\mathrm{O}$ valor esperado e a variância de $\Delta x$ são dados por:

$$
E(\Delta x)=v \Delta t
$$

$$
V(\Delta x)=\sigma^{2} \Delta t
$$

lembrando que $E(\Delta z)=0$ e $V(\Delta z)=\Delta t$

Como houve uma troca de variável de estado, a árvore binomial proposta na Figura 1 deve ser alterada a fim de exibir variações em $x$, melhor do que em $S$. Para isso, basta aplicar o logaritmo em cada nó e então a árvore passa a ser uma árvore aditiva, onde a variável $x$ move-se para cima ou para baixo segundo os incrementos $\ln u$ e $\ln d$, respectivamente. As probabilidades neutra ao risco de subida e descida não se modificam.

Analisando a distribuição de probabilidades dos valores futuros de $x$, através da média e variância de $\Delta x$, as expressões abaixo são obtidas:

$$
E(\Delta x)=p \ln u+q \ln d
$$

$$
V(\Delta x)=p q(\ln u-\ln d)
$$

Como a distribuição de probabilidades em cada período da árvore é dada por uma distribuição binomial então, quanto maior o número de intervalos de tempo, mais ela se aproxima de uma distribuição normal. Para garantir que ambas as distribuições sejam 
idênticas no limite, seus momentos devem ser iguais, ou seja, as médias e as variâncias têm de ser idênticas. Logo, a equação (9) deve ser igual à (11) e a (10) igual à (12)

$$
\left\{\begin{array}{c}
p \ln u+q \ln d=v \Delta t \\
p q(\ln u-\ln d)=\sigma^{2} \Delta t \\
p+q=1
\end{array}\right.
$$

Este sistema tem grau de liberdade um, já que existem três equações e quatro incógnitas. CRR utilizaram saltos com valores absolutos iguais para a quarta equação, ou seja, ao sistema anterior foi acrescida a equação $\ln u=-\ln d$. A solução do sistema foi encontrada assumindo que potências de $\Delta t$ superiores a unidade devem tender a zero.

$$
\begin{aligned}
& \ln u=\sigma \sqrt{\Delta t} \\
& \ln d=-\sigma \sqrt{\Delta t} \\
& p=\frac{1}{2}+\frac{1}{2} \frac{v}{\sigma} \sqrt{\Delta t}
\end{aligned}
$$

O logaritmo pode ser eliminado nas equações acima

$$
\begin{aligned}
& u=e^{\sigma \sqrt{\Delta t}} \\
& d=e^{-\sigma \sqrt{\Delta t}} \\
& p=\frac{1}{2}+\frac{1}{2} \frac{v}{\sigma} \sqrt{\Delta t}
\end{aligned}
$$

Ao assumir $\ln u=-\ln d$, ou $u=1 / d$, o modelo de CRR garante que a árvore binomial torna-se recombinante. Uma árvore binomial é recombinante quando em qualquer dois intervalos de tempo consecutivos, um movimento de subida seguido por um movimento de descida é exatamente o mesmo que um movimento de descida seguido por um movimento de subida. Essa propriedade diminui drasticamente o número de nós em cada período, a medida que $n$ cresce. Por exemplo, uma árvore recombinante com três períodos possui 4 nós no terceiro período. A Figura 1 representa árvore binomial recombinante. Caso a árvore não seja recombinante, como mostrado na Figura 2, então no terceiro período a árvore teria $2^{3}=8$ nós. Assim, uma árvore recombinante possui $i+1$ nós no i-ésimo período (o número de nós em cada intervalo de tempo cresce linearmente) e uma árvore não recombinante possui $2^{i}$ nós no $i$-ésimo período (o número de nós em cada intervalo de tempo cresce exponencialmente).

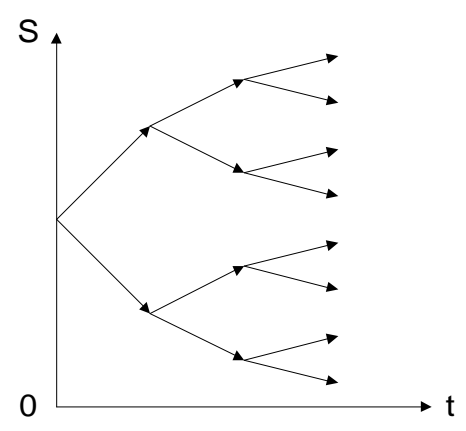

Figura 2 - Árvore Binomial não recombinante. 


\subsection{Modelo de Jarrow Rudd (JR)}

Jarrow \& Rudd (1983) propuseram que probabilidade neutra ao risco fosse dada por $p=1 / 2$. Assim, o sistema dado em (13) passa a ter duas equações e duas incógnitas. Do mesmo que o modelo de CRR, o modelo de JR supõe que potências de $\Delta t$ maiores do que a unidade tendem para zero. Os parâmetros de JR são dados a seguir:

$$
\begin{aligned}
& u=e^{\left(v-1 / 2 \sigma^{2}\right) \Delta t+\sigma \sqrt{\Delta t}} \\
& d=e^{\left(v-1 / 2 \sigma^{2}\right) \Delta t-\sigma \sqrt{\Delta t}} \\
& p=1 / 2
\end{aligned}
$$

lembrando que $v$ é dado pela equação (7). A árvore binomial gerada pelos parâmetros de JR é também recombinante.

\subsection{Modelo de Hull e White (HW)}

Um modelo mais preciso que os anteriores foi proposto por Hull \& White (1988). Nele os termos $(\Delta t)^{2}$ não foram desprezados, diferentemente dos modelos de CRR e JR. Com isso, o modelo torna-se mais preciso que os outros quando $n$ é pequeno. Os parâmetros do modelo são dados por

$$
\begin{aligned}
& u=\left[\left(m_{1}^{2}+m_{2}^{2}+1\right)+\sqrt{\left(m_{1}^{2}+m_{2}^{2}+1\right)^{2}-4 m_{1}^{2}}\right] /\left(2 m_{1}\right) \\
& d=1 / u \\
& p=\left(m_{1}-d\right) /(u-d)
\end{aligned}
$$

onde $m_{1}$ e $m_{2}$ são dados por $m_{1}=e^{r \Delta t}$ e $m_{2}^{2}=m_{1}^{2}\left(e^{\sigma^{2} \Delta t}-1\right)$.

\subsection{Modelo de Trigeorgis (TRG)}

Trigeorgis (1991) propôs um outro modelo para o cálculo dos parâmetros da árvore binomial. Através do lema de Itô, TRG chegou ao processo estocástico para a variável de estado $x=\ln S$, dado pela equação diferencial estocástica a seguir:

$$
d x=\left(i-1 / 2 \sigma^{2}\right) d t+\sigma d z
$$

Procedendo de maneira análoga ao modelo de CRR, TRG igualou a média e a variância do processo estocástico contínuo com a média e variância do processo discreto representado por uma árvore binomial aditiva. Ao sistema de equações foi também acrescida a condição que os incrementos teriam valores absolutos iguais. Neste modelo, potências de $\Delta t$ superior a unidade não foram desprezadas. Assim, foi obtido um sistema cuja solução é dada a seguir:

$$
\begin{aligned}
& \ln u=\sqrt{\sigma^{2} \Delta t+\left(v-\sigma^{2}\right)^{2} \Delta t^{2}} \\
& \ln u=-\ln d \\
& p=\frac{1}{2}+\frac{1}{2} \frac{v \Delta t}{\ln u}
\end{aligned}
$$

O modelo de TRG também seria válido se, como no modelo de JR, a probabilidade de transição fosse considerada constante e igual a $1 \frac{1}{2}$. 


\section{Avaliando Opções Americanas}

As opções americanas são caracterizadas pela possibilidade de exercício antecipado. Como dito anteriormente, opções de compra americana sobre um ativo objeto que não paga dividendos pode ser avaliada como se fosse uma opção de compra européia, ou seja o exercício antecipado não é ótimo. Já as opções de venda do tipo Americana (independente se o ativo objeto paga ou não dividendos) e opções de compra do tipo Americana, onde o ativo objeto necessariamente paga dividendos, podem ser exercidas antecipadamente.

Um grande empecilho surge quando o exercício antecipado pode ser ótimo: não existe solução analítica para tais modelos. Assim, o Modelo Binomial surge como uma aproximação para o valor verdadeiro de tais opções. Amin \& Khanna (1994) mostraram que opções americanas, quando avaliadas usando Modelo Binomial convergem para o valor verdadeiro.

Uma árvore binomial onde o período entre o lançamento da opção e seu vencimento foi dividido em $n$ intervalos de tempo possui $n+1$ nós no $n$-ésimo período. Um algoritmo para calcular o valor de uma opção usando os modelos de árvores binomiais não necessita armazenar mais do que $n+1$ nós. Os nós da árvore serão numerados como na Figura 3. O preço da ação é calculado recursivamente e o valor em cada nó é dado por $S u^{\mathrm{j}} d^{\mathrm{n}-\mathrm{j}}$. O uso da recursividade é um artifício de programação muito importante, que nesse caso irá reduzir a complexidade do algoritmo, pois evita que operações mais complexas que a multiplicação (potenciação) sejam utilizadas.

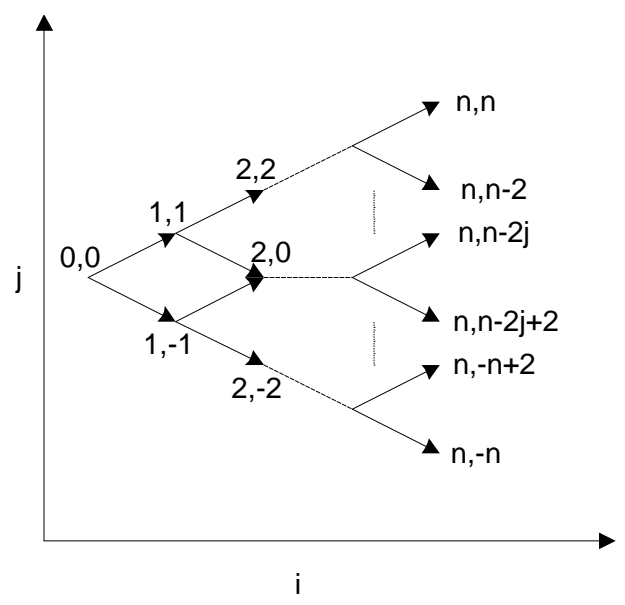

Figura 3 - Numeração dos Nós de uma Árvore Binomial

A rotina de programação dinâmica é inicializada estabelecendo, por exemplo, o preço da opção de compra no instante T é $C_{T}=\max \left(S_{T}-K, 0\right)$ em cada um dos nós terminais, onde $K$ é o preço de exercício da opção (caso a opção fosse de venda, o preço da opção nos nós terminais seria $C_{T}=\max \left(K-S_{T}, 0\right)$. Considere o nó no alto à direita da Figura $1, C_{T}\left(u^{3} S\right)$ é estabelecido para $\max \left(u^{3} S-K, 0\right)$. No nó anterior correspondente ao preço da ação $u^{2} S$ no tempo $T-\Delta t$, o valor da opção de compra $C_{T-\Delta t}\left(u^{2} S\right)$ é estabelecido para

$$
\max \left\{\max \left(\left(\mathrm{u}^{2} S-K\right), 0\right), e^{-r \Delta t}\left[p C_{T}\left(u^{3} S\right)+(1-p) C_{T}\left(u^{2} d S\right)\right]\right\}
$$


Isto é, o valor da opção de compra do tipo Americana é o máximo entre o valor do exercício imediato e o valor presente de continuação. O valor da opção de compra nos nós restantes são calculados de maneira similar utilizando recursividade.

Um cuidado que deve ser tomado ao implementar o algoritmo é que não sejam feitas multiplicações desnecessárias repetidas vezes. Para isso, deve-se verificar com antecedência o que é constante ao longo do algoritmo. Essas constantes serão pré calculadas e, deste modo, serão eliminadas operações redundantes, principalmente dentro dos loops. No apêndice, será apresentado o algoritmo para avaliação de opções americanas utilizando o modelo CRR.

\section{Convergência dos Modelos Binomiais}

Nesta seção serão apresentadas algumas soluções propostas na literatura para acelerar a convergência dos modelos binomiais. Todos os gráficos são de uma opção de compra do tipo Americana cujo preço do ativo objeto $(S)$ é $\$ 110,00$, o preço de exercício $(K)$ é $\$ 100,00$, o tempo de maturação $(T)$ é igual a 6 meses, a volatilidade do preço da ação $(\sigma)$ é 30\%a.a., a taxa de dividendos $(\delta)$ é $3 \%$ a.a. e a taxa de juros livre de risco $(r)$ é $7 \%$ a.a..

Quando métodos numéricos são utilizados para resolver problemas, é comum verificar se o algoritmo empregado converge para o conjunto de soluções e quão rápido ele o faz. A convergência teórica é uma propriedade altamente desejável para algoritmos numéricos. Se dois algoritmos são convergentes, mas o primeiro converge mais rápido do que o segundo, então o primeiro será selecionado.

No cálculo do preço de uma opção baseado em métodos de árvores binomiais, a definição do números de intervalos de tempo, entre o lançamento e a maturidade da opção, é fundamental para a que o modelo consiga convergir para o valor verdadeiro, mas a convergência é lenta e oscilatória. Esta característica pode ser confirmada no gráfico da Figura 4, que apresenta a convergência dos modelos de árvores binomiais apresentados seção 2. Observe que o padrão de convergência é praticamente o mesmo para os quatro modelos apresentados.

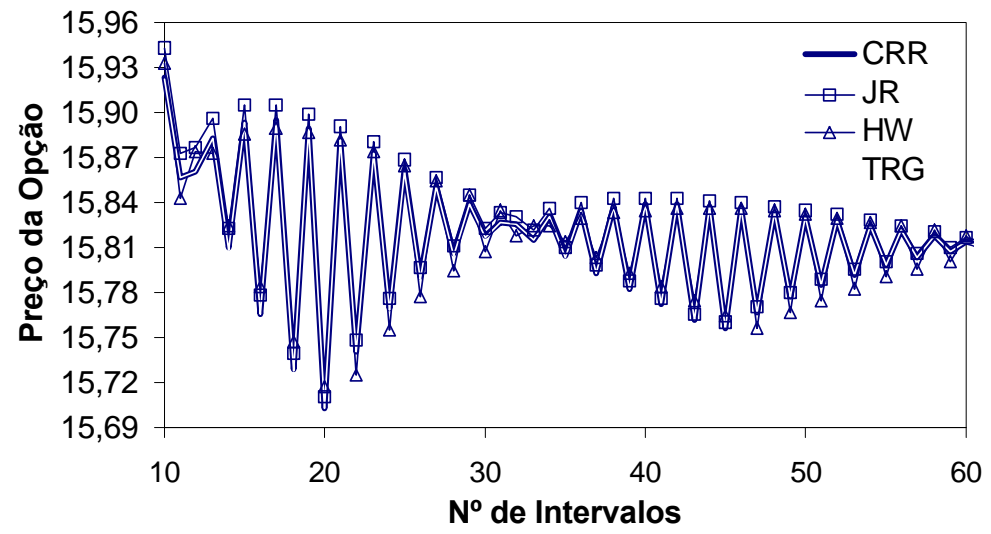

Figura 4 - Gráfico de convergência dos modelos CRR, JR, HW e TRG

Nos itens a seguir serão apresentados os principais métodos para acelerar a convergência dos modelos de árvores binomiais. 


\subsection{Método dos Valores Médios (MVM)}

Muitas vezes, regras práticas são utilizadas para tirar vantagens de algumas características de modelos numéricos. No caso das árvores binomiais, a média entre os valores obtidos nos passos $n+1$ e $n$ é calculada a fim de tomar vantagem da regra de convergência oscilatória. O gráfico da Figura 5 ilustra a convergência do MVM comparado ao modelo de CRR.

Broadie \& Detemple (1996) afirmaram, seguindo os resultados obtidos em seus modelos de simulação, que este método não possui performance significativamente melhor do que os modelos binomiais tradicionais. Já Mayhew (1998) mostrou, usando simulações similares àquelas feitas por Broadie \& Detemple, que o Método dos Valores Médios sugere uma velocidade duas vezes maior do que os modelos tradicionais para um mesmo nível de precisão. O gráfico da Figura 5 sugere que a convergência do MVM seja superior ao modelo CRR.

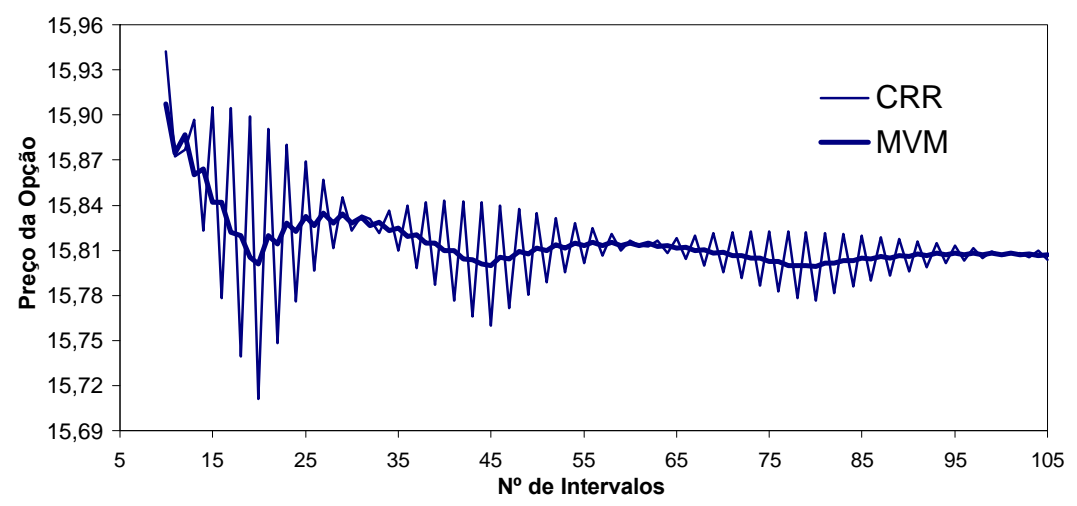

Figura 5 - Gráfico ilustra a convergência do modelo MVM comparado ao modelo CRR

\subsection{Método Binomial Black e Scholes (BBS)}

Este método foi proposto por Broadie \& Detemple (1996) e consiste em substituir a fórmula do valor de continuação (apresentada a seguir) no passo $n-1$ pela fórmula de Black e Scholes.

$$
e^{-r \Delta t}[p C(i+1)+(1-p) C(i-1)], \quad i=-n+1,-n+2, \mathrm{~K}, n-1
$$

O trabalho computacional para calcular o valor de continuação em cada nó é de somente duas multiplicações. Assim, a computação da fórmula de Black e Scholes requer a avaliação de uma função de distribuição normal cumulativa duas vezes para a cada nó. Mas, como este cálculo é feito somente em $n$ nós, o tempo de processamento é praticamente o mesmo que aquele necessário para calcular uma opção usando o modelo binomial de CRR. Para que a complexidade do modelo binomial não aumente, métodos eficientes para o cálculo da função de distribuição normal cumulativa devem ser utilizados. Abramowitz \& Stegun (1972) e Moro (1995) implementaram algoritmos eficientes que podem ser utilizados. O gráfico da Figura 6 exibe a convergência dos modelos BBS e CRR. Repare que a convergência do BBS deixou de ser oscilatória para ser mais suave, e também sua precisão é maior quando comparado a um mesmo número de intervalos de tempo. 


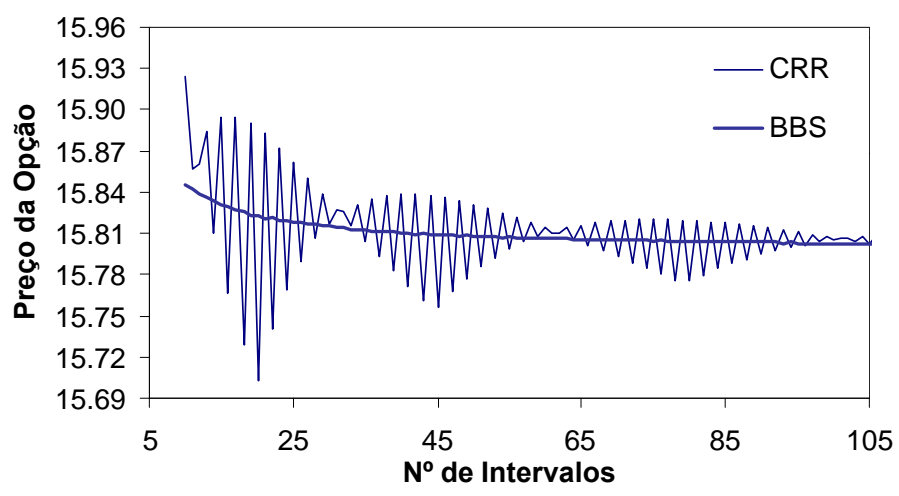

Figura 6 - Gráfico de convergência dos modelos BBS e CRR

\subsection{Modelo BBS com Extrapolação de Richardson (BBSR)}

Segundo Broadie \& Detemple (1996), a convergência mais suave obtida com BBS sugere a utilização da Extrapolação de Richardson. Assim, o método BBSR adiciona uma extrapolação de dois pontos diretamente sobre o número de intervalos de tempo do modelo BBS. Para calcular o preço de uma opção com $n$ intervalos de tempo usando o método BBSR, três passos devem ser seguidos:

- calcular o preço de uma opção usando o modelo BBS com $n$ intervalos de tempo $\left(C_{n}\right)$;

- calcular o preço de uma opção usando o modelo BBS com $m=n / 2$ intervalos de tempo $\left(C_{m}\right)$;

- usar a fórmula de extrapolação $2 C_{n}-C_{m}$.

Broadie \& Detemple mostraram que o modelo BBSR é significativamente melhor do que qualquer outro método do tipo binomial. O gráfico da Figura 7 compara os modelos de CRR, BBS e BBSR. Este gráfico já sugere uma performance melhor em termos de convergência do modelo BBSR. Para se ter uma idéia, o BBSR com $n=100$ é tão preciso quanto o CRR com $n=1000$ e cerca de 55 vezes mais rápido. Esta conclusão pode ser encontrada no artigo de Broadie \& Detemple. Utilizando distribuição de probabilidades para cada um dos parâmetros da opção e também uma amostra relativamente grande de opções eles traçaram gráficos de Velocidade Computacional por Erro Médio Quadrático. Em todos esses gráficos o modelos BBSR apresentou a melhor performance dentre todos os modelos binomiais apresentados.

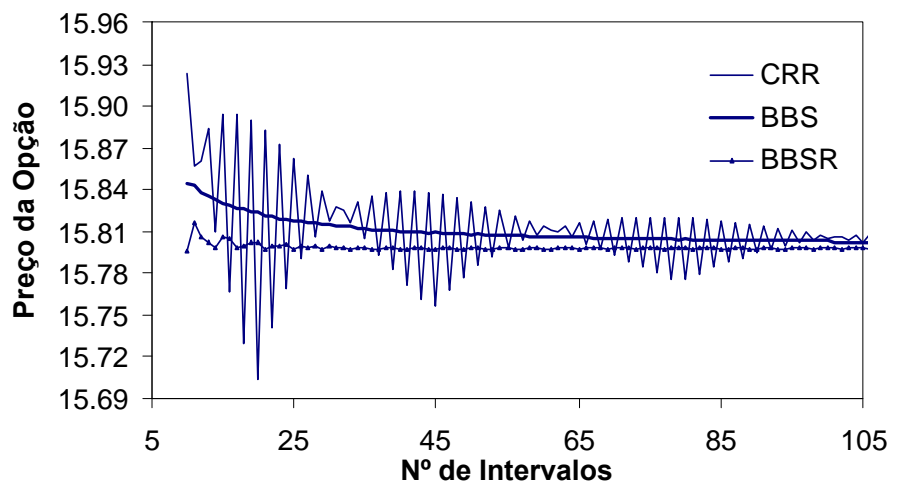

Figura 7 - Gráfico de convergência dos modelos BBSR, BBS e CRR 
O gráfico da Figura 8 ilustra as regras de convergências dos modelos CRR, MVM, BBS e BBSR.

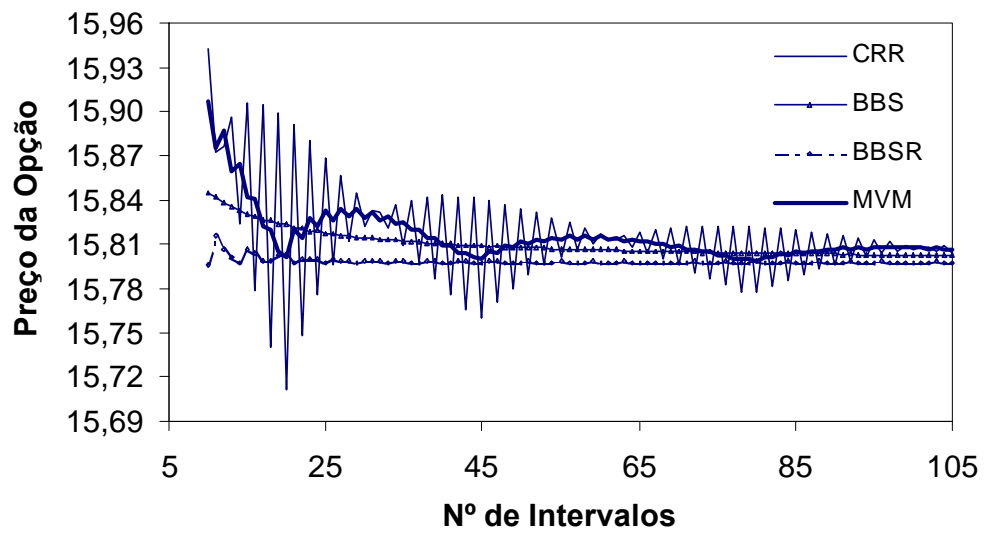

Figura 8 - Gráfico de convergência dos modelos CRR, BBS, BBSR e MVM

Existem outros métodos para reduzir os efeitos da convergência oscilatória e/ou acelerar a velocidade de convergência dos modelos binomiais. Leisen \& Reimer (1995) sugerem que o sistema dado em (13) seja completado com uma equação que posiciona o preço de exercício no centro da árvore para qualquer nível de discretização. Este método praticamente elimina a forma oscilatória, mas não consegue acelerar a velocidade de convergência. Outro método também apresentado por Leisen \& Reimer (1995) utiliza uma aproximação normal para a distribuição binomial.

\section{Conclusão}

Neste trabalho foram apresentados os principais modelos numéricos para avaliação de opções usando árvores binomiais e algumas técnicas de implementação.

Três métodos propostos na literatura para acelerar a convergência dos modelos binomiais foram analisados: Método do Valores Médios (MVM), Método Binomial Black Scholes (BBS) e Método BBS com Extrapolação de Richardson.

Uma maneira simples, mas eficaz de reduzir os efeitos da convergência oscilatória é tomar a média entre os valores de dois passos consecutivos de uma árvore binomial simples (MVM). A mistura de um modelo numérico com uma fórmula analítica (BBS) propiciou um aumento na performance do modelo de árvore binomial. Quando comparado a uma árvore binomial pura, a forma oscilatória foi praticamente eliminada e a convergência tornou-se mais rápida. Utilizando-se extrapolação de Richardson, foi possível incrementar a performance do modelo BBS. Gráficos de convergência sugeriram que os métodos propostos são mais precisos e convergem mais rapidamente que os modelos tradicionais, para um mesmo nível de discretização.

Dos três métodos apresentados, o método BBS com Extrapolação de Richardson sugere uma melhor performance, ou seja, este método necessitaria de um número menor de passos para atingir um resultado tão preciso quanto os outros métodos apresentados. 


\section{Apêndice}

Neste apêndice será apresentado apenas o algoritmo para implementação computacional do Modelo Binomial usando os parâmetros de CRR. Para implementar os modelos de JR, HW e TRG basta substituir o cálculo dos parâmetros no algoritmo por aqueles apropriados.

Para implementar o BBS, deve-se escolher um dos modelos para o cálculo dos parâmetros da árvore binomial. Dentro do procedimento de programação dinâmica, deve-se verificar os nós que pertencem ao penúltimo período e a fórmula de Black e Scholes deve ser usada para avaliar o preço da opção em cada um desses nós. A fórmula de Black e Scholes utiliza um algoritmo para cálculo da distribuição normal acumulada. Abramowitz \& Stegun (1972) e Moro (1995) sugerem algoritmos precisos e rápidos para o cálculo da distribuição normal acumulada.

Já o modelo BBSR é o mais fácil de ser implementado, pois a extrapolação é feita sobre o número de intervalos de tempo. A fórmula $C=2 C_{n}-C_{m}$ deve ser computada, onde $C$ é o preço BBSR da opção com $n$ intervalos de tempo, $C_{n}$ é o preço BBS com $n$ intervalos de tempo e $C_{m}$ é o preço BBS com $m=n / 2$ intervalos de tempo.

\subsection{Modelo de Cox, Ross e Rubinstein}

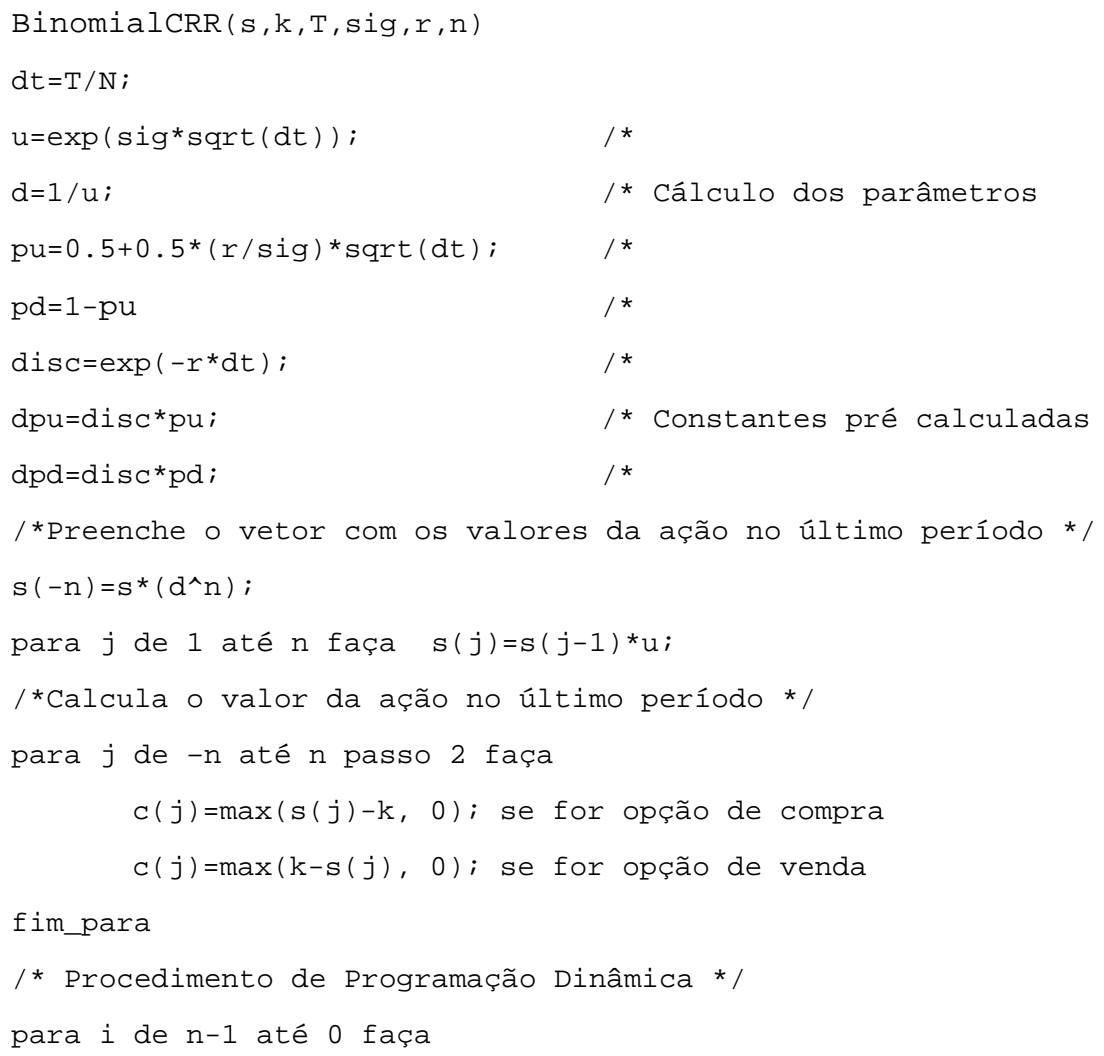




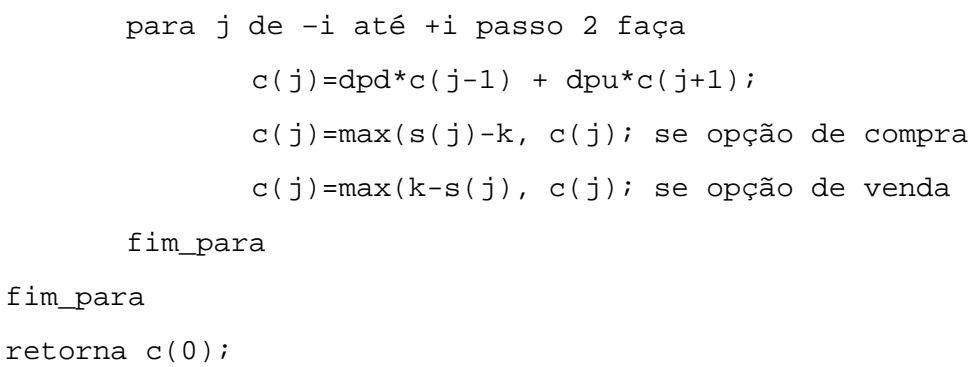

\section{Referências Bibliográficas}

(1) Abramowitz, W. \& Stegun, I. (1972). Handbook of Mathematical Functions. Dover Publications.

(2) Amin, K \& Khanna, A. (1994). Convergence of American Option Values from Discrete to Continuous Time Financial Models. Mathematical Finance, 4, 289-304.

(3) Black, F. \& Scholes, M. (1973). The Price of Options and Corporate Liabilities. Journal of Political Economy, 81, 637-659.

(4) Brennan, M. \& Schwartz, E.S. (1978). Finite Difference Methods and the Jump Process Arising in the Pricing of Contingent Claims: A Synthesis. Journal of Financial and Quantitative Analysis, 20, 461-473.

(5) Broadie, M. \& Detemple, J. (1996). American Option Valuation: New Bounds, Approximations, and a Comparison of Existing Methods. Review of Financial Studies, 9, 1211-1250.

(6) Broadie, M. \& Detemple, J. (1997). "Recent Advances in Numerical Methods for Pricing Derivative Securities", in Numerical Methods in Finance. Cambridge University Press, Cambridge.

(7) Chriss, N. (1995). Black-Scholes and Beyond: Option Pricing Models. McGraw-Hill, Nova York.

(8) Clewlow, L. \& Strickland, C. (1998). Implementing Derivatives Models. John Wiley \& Sons, Nova York.

(9) Cox, J. \& Ross S. (1976). The Valuation of Options for Alternative Stochastic Process. Journal of Financial Economics, 3, 145-166.

(10) Cox, J.; Ross, S. \& Rubinstein, M. (1979). Option Pricing: A Simplified Approach. Journal of Financial Economics, 7, 229-263.

(11) Hull, J.C. \& White, A. (1988). The Use of the Control Variate Technique in Option Pricing. Journal of Financial and Quantitative Analysis, 23, 237-251.

(12) Hull, J.C. (1997). Options, Futures and Other Derivatives. Prentice Hall, Englewood Cliffs, Nova Jersey.

(13) Jarrow, R. \& Rudd, A. (1983). Option Pricing. Dow Jones- Irwin.

(14) Leisen, D.P.J. (1998). Pricing the American put option: A detailed convergence analysis for binomial models. Journal of Economic Dynamics \& Control, 22, 1419-1444. 
(15) Leisen, D.P.J. \& Reimer, M. (1995). Binomial Models for Option Valuation: Examining and Improving Convergence. Discussion Paper, B-309, University of Bonn.

(16) Mayhew, S. (1998). Numerical Results on the Convergence of Binomial Option Pricing Model. Working Paper, Krannert School of Management, Purdue University.

(17) Merton, R.C. (1973). The Theory of Rational Option Pricing. Bell Journal of Economics and Management Science, 4, 141-183.

(18) Moro, B. (1995). Fast Computation of Cumulative Normal Distribution Function. Working Paper, TMG Financial Products, Greenwich, Connecticut.

(19) Trigeorgis, L. (1991). A Log-Transformed Binomial Numerical Analysis Method for Valuing Complex Multi-Option Investments. Journal of Financial and Quantitative Analysis, 26, 309-326. 